\title{
USO DE ALGUNOS PARÁMETROS INDICADORES MICROBIOLÓGICOS Y BIOQUÍMICOS PARA LA EVALUACIÓN DE LA CONTAMINACIÓN POR HIDROCARBUROS Y LA BIODEGRADACIÓN DE LOS MISMOS, EN LA ZONA DEL LAGO TITIKAKA (SAN PEDRO DE TIQUINA, BOLIVIA)
}

\section{SOME BIOLOGICAL AND BIOCHEMICAL INDICATOR PARAMETERS TO EVALUATE HYDROCARBON CONTAMINATION AND BIODEGRADATION AT TITIKAKA'S LAKE ZONE (SAN PEDRO DE TIQUINA, BOLIVIA)}

\author{
Francisco Fontúrbel Rada ${ }^{1}$
}

\begin{abstract}
Resumen
Se estudió la variación en el tiempo de la biomasa microbiana, el pH y la actividad enzimática (para proteasas, ureasas y sacarasas), en suelos del municipio de San Pedro de Tiquina, expuestos naturalmente a pequeñas vertientes de hidrocarburos. Se estudiaron muestras de época seca y de época de lluvias. La biomasa presentó un descenso hasta llegar a $0 \mathrm{meqC} / \mathrm{kg}$ en época seca en la muestra tratada con hidrocarburos, las muestras de época de lluvias mostraron un comportamiento exponencial, en ambos casos. El análisis de $\mathrm{pH}$ mostró una tendencia a regularse en un rango de 5.50-5.60 a las dos semanas de exposición, para ambas épocas. Los análisis de actividad enzimática mostraron un comportamiento de tipo recíproco y polinomial de $2^{\circ}$ grado para proteasas, sacarasas y ureasas, aunque se vio que las sacarasas no tienen sensibilidad a este tipo de contaminación. Los análisis de varianza efectuados mostraron diferencia significativa entre los tratamientos con hidrocarburos y los testigos. Los análisis de correlación de Pearson mostraron una correlación regular entre pH-biomasa y biomasa-proteasas, y una alta correlación entre proteasas-ureasas.

Se analizaron los datos y se determinó que la conjunción de estos parámetros puede ser utilizada como un indicador para evaluar la contaminación y la potencialidad de biodegradación natural e in-situ de hidrocarburos en suelos de la zona del lago Titikaka.
\end{abstract}

Palabras clave: Hidrocarburos, ecosistema edáfico, biodegradación, biomasa microbiana, actividad enzimática.

\begin{abstract}
Microbial biomass, $\mathrm{pH}$ and enzymatic activity (for proteases, sacharases and ureases) time variation were studied in naturally hydrocarbon exposed soils at the municipality of San Pedro de Tiquina. Dry and wet season samples were analyzed. For dry season samples, the microbial biomass on hydrocarbon treated soil reach a $0 \mathrm{meqC} / \mathrm{kg}$ value, whereas wet season samples show an exponential behavior for control and treated samples. In both seasons, $\mathrm{pH}$ analysis shows a regulation tendency to values of a 5.50-5.60 range at two weeks of exposure. Enzymatic activity analysis shows a reciprocal and $2^{\text {nd }}$ grade polynomial behavior for proteases, sacharases and ureases. Also sacharases activity was observed that is not sensitive for this kind of contamination. Variance analysis performed shows significant differences between control and hydrocarbontreated samples. Pearson correlation analysis shows a medium relationship between biomass-pH and biomass-proteases, and a high correlation between proteases-ureases.

Results suggest that a conjunction of these parameters could be used as an indicator to evaluate hydrocarbon contamination and in-situ natural biodegradation potentialities in Titikaka's lake zone soils.
\end{abstract}

Keywords: Hydrocarbons, edaphic ecosystem, biodegradation, microbial biomass, enzymatic activity.

\section{Introducción}

En la actualidad, la contaminación por hidrocarburos es una de las principales causas de destrucción de los ecosistemas edáficos (Fontúrbel \& Achá, 2003), ya que el uso masivo y el transporte transfronterizo tanto de petróleo crudo como de sus derivados, hace que los derrames de hidrocarburos sean cada vez más frecuentes. La composición química de los hidrocarburos es muy variable: en la naturaleza normalmente se encuentran como mezclas de diferentes especies moleculares, que en mayor o menor grado afectan a la flora, a la fauna y a los microorganismos del suelo (Madigan et al., 1999).

La fertilidad de los suelos es un factor muy importante para el crecimiento de las plantas, y por lo tanto para la existencia y sobrevivencia de los animales, que se alimentan de éstas (Krebs, 1985). Los microorganismos del suelo (tanto hongos como 
bacterias) determinan en gran parte la fertilidad del suelo por la mediación de los procesos de descomposición de la materia orgánica, la movilización de nutrientes y la capacidad de intercambio catiónico (Smith \& Smith, 2001). Es por esta razón que el estudio de los microorganismos del suelo es fundamental para su conservación y manejo adecuado.

Varios procesos bioquímicos y ecológicos propios del suelo se desestabilizan por la acción de los hidrocarburos, ya que sus efectos tóxicos afectan a casi todas las especies del ecosistema edáfico en mayor o menor grado, de forma directa o indirecta (Madigan et al., 1999). A pesar que existen muy pocos estudios sobre el tema contextualizados a la propia realidad, los suelos bolivianos no son la excepción a la regla.

De acuerdo con Varnam \& Evans (2000) prácticamente todos los ecosistemas edáficos $\mathrm{y}$ acuáticos del planeta poseen -en mayor o menor proporción- cepas de microorganismos capaces de responder naturalmente a los problemas de contaminación por medio de la biodegradación de los contaminantes, incluyendo a los hidrocarburos. Si bien estos procesos se dan de forma natural, la reducida cantidad de microorganismos hace que los tiempos de degradación sean demasiado largos como para observar un resultado que pueda ser considerado como positivo. Sin embargo, existen técnicas de estimulación-denominadas en su conjunto bioremediación- capaces de reducir significativamente el tiempo de degradación del contaminante estimulando el crecimiento de la comunidad de microorganismos degradadores (sensu Solano-Serena et al., 2000).

A raíz del derrame de petróleo del año 2000 en los departamentos de La Paz y Oruro, ocasionado por el deficiente estado de los oleoductos de la empresa Transredes, Bolivia percibió que no se cuenta con información básica sobre el tema de contaminación por hidrocarburos e indicadores que permitan hacer una evaluación de los daños producidos por este tipo de desastres ecológicos y dar una perspectiva de solución por medio de técnicas de biodegradación natural in-situ.

Por estas razones se decidió estudiar la problemática de la contaminación por hidrocarburos en suelos afectados de la zona del lago Titikaka, correspondientes al municipio de San Pedro de Tiquina (Fontúrbel \& Achá, 2003). En el presente trabajo se describen y analizan algunos de los parámetros microbiológicos y bioquímicos, susceptibles de ser usados como indicadores para evaluar el impacto de la contaminación por hidrocarburos y la potencialidad de degradación natural in-situ de los mismos en esta zona, y por extrapolación, en el altiplano boliviano.

\section{Materiales y métodos \\ Área de estudio}

El sitio de estudio se encuentra en el municipio de San Pedro de Tiquina (Departamento de La Paz, Bolivia), a menos de $2 \mathrm{~km}$ del estrecho de Tiquina, y a $180 \mathrm{~m}$ de la orilla del lago. Las coordenadas geográficas del sitio en UTM son 0525972 / 8210582 (16 $16^{\circ}$ '53.10' 'S, 68 45' 19.12' 'W), y la altitud del sitio es de $3845 \mathrm{~m}$. Este sitio fue escogido por la presencia natural de pequeñas vertientes de hidrocarburos, ya que según Parrish et al. (1999), una exposición constante a los hidrocarburos favorece la presencia y abundancia de los microorganismos resistentes y/o degradadores de hidrocarburos.

\section{Muestreo}

Se empleó el protocolo de muestreo de suelos en capa arable propuesto por Totsche (1995), tomando muestras aleatorias a $30 \mathrm{~cm}$ de profundidad, y realizando una muestra mixta por cuarteo. En el laboratorio las muestras de suelo fueron tamizadas con una malla de $2 \mathrm{~mm}$. Se tomaron muestras en época seca (Octubre de 2000) y en época de lluvias (Febrero de 2001). El suelo no se esterilizó ni recibió otro tipo de tratamiento post-muestro para mantener sus condiciones naturales (Duarte \& Ferreira, 2000).

Determinación de parámetros fisicoquímicos iniciales de referencia

El pH inicial de la muestra fue determinado in-situ mediante el método descrito por Morales et al. (1997). En laboratorio se determinó la materia orgánica según el método descrito por Forster (1995) y la textura fue determinada por el método de tamices descrito por Chilón (1996).

\section{Diseño de los tratamientos}

Con el suelo tamizado, se elaboraron tratamientos para el suelo correspondiente a la época seca y a la época de lluvias, por medio de la adición de diferentes concentraciones de aceite de motor sin quemar. Los intervalos de tiempo usados para las mediciones se indican en las respectivas gráficas, puesto que se emplearon diferentes escalas de tiempo, según el tipo de análisis. Para todas las pruebas se utilizó un factor de corrección de humedad relativa

\section{Determinación de la biomasa microbiana y pH}

Se empleó el método de fumigación-extracción por cloroformo aplicado a suelos contaminados con petróleo (Fontúrbel \& Achá, 2002), basado en los protocolos de Jenkinson \& Powlson (1976) y Tate et al. (1988). Se aplicó el método para determinación de miliequivalentes de carbono por $\mathrm{kg}$ de suelo. El $\mathrm{pH}$ en laboratorio se determinó de acuerdo al método descrito por Morales et al. (1997).

\section{Determinación de la actividad enzimática}

Para estudiar la actividad enzimática de tres enzimas: proteasas, sacarasas y ureasas, se emplearon metodologías simplificadas aplicadas a suelos 


\section{INDICADORES DE CONTAMINACIÓN Y BIODEGRADACIÓN DE HIDROCARBUROS EN SUELOS DEL LAGO TITIKAKA}

Diciembre 2004

contaminados por hidrocarburos, según Fontúrbel (2002).

\section{Análisis estadísticos}

Se realizaron análisis de regresión lineal mediante el software Regression Analyzer 3.2, de análisis de varianza (ANOVA) mediante el software ESBStats Standard 1.2. Los valores $F$ tabulados fueron obtenidos de Daniel (1996). Los análisis de correlación se elaboraron mediante el software ESBStats Standard 1.2. Todas las muestras se trabajaron por triplicado.

\section{Resultados}

\section{Parámetros iniciales del suelo}

En las muestras de época seca, se determinó que el $\mathrm{pH}$ del suelo era de 4.79 , la humedad relativa del $14.44 \%$, la textura era arcillosa, la materia orgánica de $3945 \mathrm{meqC} / \mathrm{g}$ y el valor inicial de biomasa microbiana era de $57.81 \mathrm{mEqC} / \mathrm{kg}$. Para la época de lluvias, el $\mathrm{pH}$ medido fue de 6.11 , la humedad relativa de $15.34 \%$, la textura arcillosa, la materia orgánica $11500 \mathrm{meqC} / \mathrm{g}$ y la biomasa microbiana inicial de $168.53 \mathrm{meqC} / \mathrm{kg}$.

\section{Biomasa microbiana}

Los resultados de biomasa microbiana en de época seca se muestran en la Figura 1, en la que se incluyen además curvas de tendencia (por regresión lineal) con los coeficientes $\mathrm{R}^{2}$ respectivos. En esta gráfica se puede apreciar que el descenso de la biomasa microbiana es paulatino tanto para el control como para la muestra contaminada, pero a partir de los 15 días de exposición, el descenso de la biomasa en la muestra contaminada es mucho más pronunciado hasta llegar a cero a los 30 días. En la Figura 2 se muestran los resultados de biomasa microbiana correspondientes a la época de lluvias, observándose en esta gráfica que los diferentes tratamientos no responden a función de carácter lineal como en el caso anterior, sino que se observa un decremento inicial, seguido de un incremento a las 24 días, y un nuevo descenso a partir de los 40 días de medición.

\section{Variación del pH}

La figura 3 muestra el cambio de los valores de $\mathrm{pH}$ para la época seca y la figura 4 para la época de lluvias. Como se puede observar en la Figura 3, a los 15 días de tratamiento se observa un notable incremento de los valores de $\mathrm{pH}$ hasta valores cercanos a 5.50, para posteriormente descender nuevamente. En el caso de las muestras de época de lluvias se observa un comportamiento inverso, donde a los 40 días de observación se da un fuerte descenso, seguido de un incremento a los 50 días. Nótese que en ambos casos, a los 15 días de tratamiento, los valores de $\mathrm{pH}$ se manejan en el rango de 5.50 a 5.60.

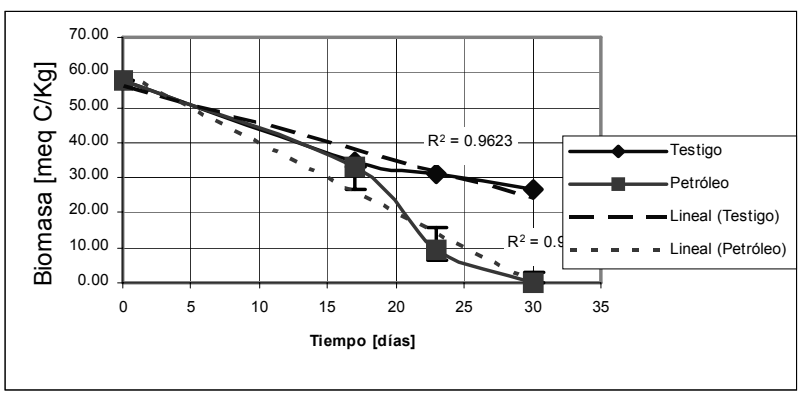

Fig. 1. Variación de la biomasa microbiana en función al tiempo para la muestra testigo y la muestra tratada con petróleo (con concentración de $25 \mathrm{ml} / 100 \mathrm{~g}$ ). Datos para época seca. La desviación estándar sobre el promedio se indica con las barras verticales.

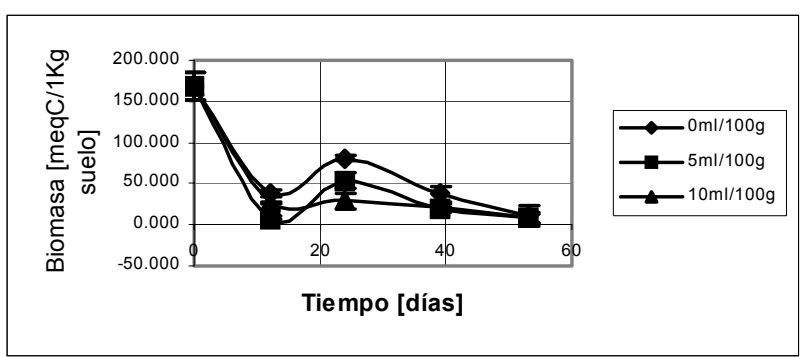

Fig. 2. Variación de la biomasa microbiana en función al tiempo para la muestra testigo y las muestras tratadas para las concentraciones de 0.5 y $10 \mathrm{ml} / 100 \mathrm{~g}$ de suelo. Datos para época de lluvias. La desviación estándar sobre el promedio se indica con las barras verticales.

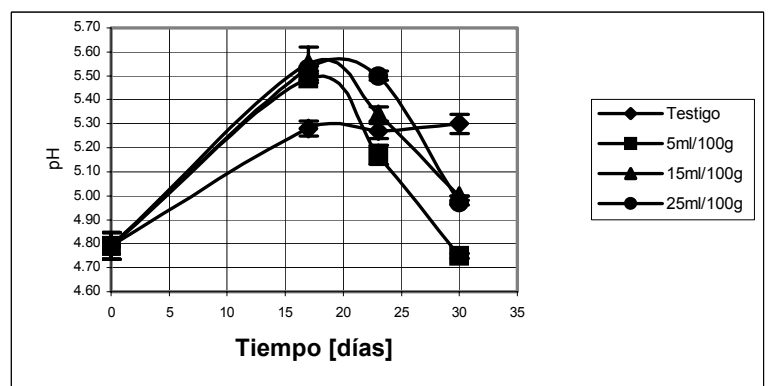

Fig. 3: Variación del pH en función al tiempo para la muestra testigo $\mathrm{y}$ las muestras tratadas con hidrocarburos para las concentraciones que se indican. Datos para época seca. La desviación estándar sobre el promedio se indica con las barras verticales.

\section{Actividad enzimática: proteasas, sacarasas y ureasas \\ Empleando las metodologías simplificadas y los} criterios expuestos en Fontúrbel (2002) se obtuvieron datos de variación de actividad enzimática en función al tiempo, para proteasas, sacarasas y ureasas.

La curva obtenida para proteasas (Figura 5) muestra un notable incremento inicial de la actividad enzimática, seguida de un descenso pasados 20 días de 
exposición (pico máximo registrado de actividad enzimática), manteniéndose la actividad enzimática a un nivel superior al inicial hasta el final de la observación. En cuanto a las sacarasas (Figura 6) se observa un comportamiento marcadamente diferente para los 3 tratamientos a los 20 días seguido de una caída brusca. Para las sacarasas (Figura 7) se observa un incremento inicial seguido de un descenso para la muestra testigo y un descenso inicial seguido de un incremento después de los 30 días para las muestras tratadas con diferentes concentraciones de hidrocarburos.

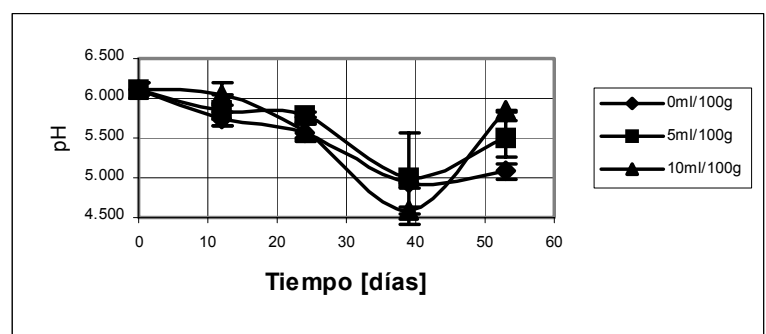

Fig. 4. Variación del pH en función al tiempo para la muestra testigo $\mathrm{y}$ las muestras tratadas con hidrocarburos para las concentraciones que se indican. Datos para época de lluvias. La desviación estándar sobre el promedio se indica con las barras verticales.

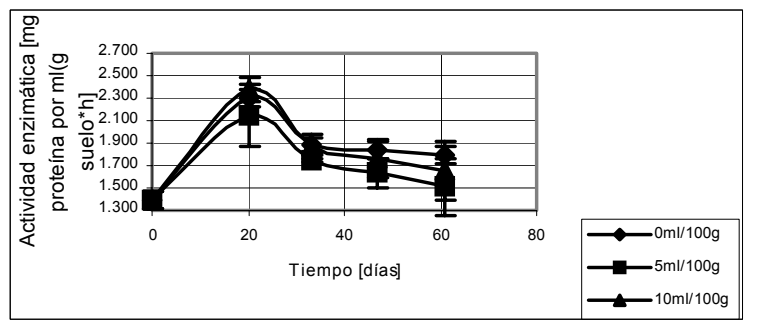

Fig. 5. Variación de la actividad enzimática de las proteasas en función al tiempo para la muestra testigo y las muestras tratadas con hidrocarburos para las concentraciones que se indican. Datos para época de lluvias. La desviación estándar sobre el promedio se indica con las barras verticales.

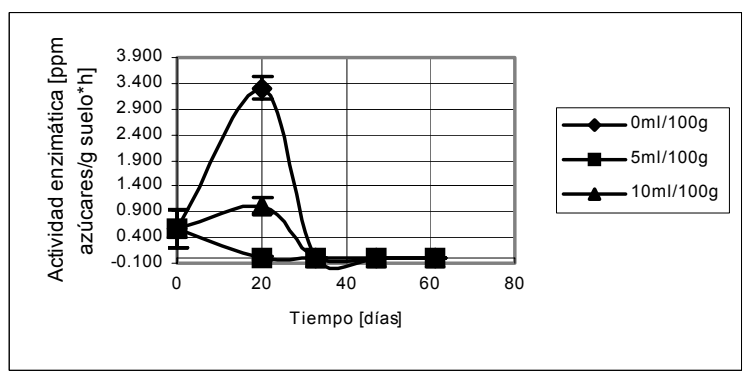

Fig. 6: Variación de la actividad enzimática de las sacarasas en función al tiempo para la muestra testigo y las muestras tratadas con hidrocarburos para las concentraciones que se indican. Datos para época de lluvias. La desviación estándar sobre el promedio se indica con las barras verticales.

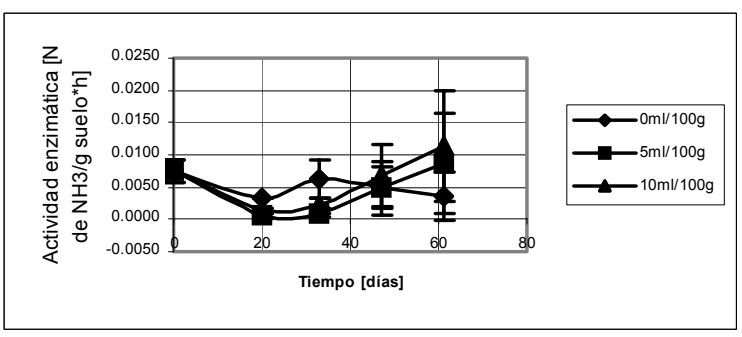

Fig. 7. Variación de la actividad enzimática de las ureasas en función al tiempo para la muestra testigo y las muestras tratadas con hidrocarburos para las concentraciones que se indican. Datos para época de lluvias. La desviación estándar sobre el promedio se indica con las barras verticales.

\section{Análisis estadísticos de los resultados}

Para las muestras de biomasa correspondientes a la época seca, el análisis de regresión mostró un comportamiento lineal, con mayor bondad de ajuste para la muestra testigo. El ANOVA realizado entre los datos del testigo y la muestra tratada mostraron que existe una diferencia significativa en la cantidad de carbono a partir del día 30. Para las muestras de época de lluvias, el análisis de regresión dio un coeficiente de 0.86 y 0.88 para el testigo y la muestra $10 \mathrm{ml} / 100 \mathrm{~g}$ respectivamente, bajo el modelo exponencial, la muestra de $5 \mathrm{ml} / 100 \mathrm{~g}$ dio un coeficiente de 0.70 para el modelo lineal. El ANOVA mostró que existe una diferencia significativa entre los resultados, en todos los tiempos.

Para las muestras de $\mathrm{pH}$ correspondientes a la época seca, el análisis de regresión dio un coeficiente de 0.92 para el testigo bajo el modelo lineal, los demás tratamientos no se ajustaron a ningún modelo de regresión. El ANOVA mostró que existe una diferencia significativa entre los resultados en todos los tiempos. Para la época de lluvias, el análisis de regresión dio un coeficiente de 0.93 para el testigo, de 0.77 para el tratamiento $5 \mathrm{ml} / 100 \mathrm{~g}$ y de 0.50 para $10 \mathrm{ml} / 100 \mathrm{~g}$, todos bajo el modelo lineal. El ANOVA mostró que existe diferencia significativa en todos los tiempos.

Las curvas de actividad enzimática de proteasas ajustaron medianamente al modelo recíproco, y las de sacarasas y ureasas al modelo polinomial de $2^{\circ}$ grado y recíproco. Los ANOVA realizados mostraron que para proteasas y sacarasas existe una diferencia significativa durante todo el tiempo de observación, mientras que para las sacarasas existe diferencia significativa solamente entre los días 12 y 29 del tratamiento, ya que luego de la caída brusca de los valores de actividad enzimática todas las muestras reportaron valores muy próximos a cero. 


\section{INDICADORES DE CONTAMINACIÓN Y BIODEGRADACIÓN DE HIDROCARBUROS EN SUELOS DEL LAGO TITIKAKA}

Diciembre 2004

Los análisis de correlación de Pearson elaborados para los datos de la época de lluvias se muestran en la Tabla 1. No se realizó esta correlación con los datos de la época seca por no contar con información de actividad enzimática.

Tabla 1. Análisis de correlación de Pearson para los datos obtenidos en época de lluvias. El criterio de calificación de coeficientes corresponde a Daniel (1996).

\begin{tabular}{|l|c|c|}
\hline Factores de relación & $\begin{array}{c}\text { Coeficiente de } \\
\text { Pearson }\end{array}$ & Calificación \\
\hline pH-biomasa & 0.555947418 & Regular correlación \\
\hline pH-proteasas & 0.050531245 & No existe correlación \\
\hline pH-sacarasas & 0.316997642 & Baja correlación \\
\hline pH-ureasas & 0.012458038 & No existe correlación \\
\hline biomasa-proteasas & 0.571478181 & Regular correlación \\
\hline biomasa-sacarasas & 0.136409331 & Baja correlación \\
\hline biomasa-ureasas & 0.319641992 & Baja correlación \\
\hline proteasas-sacarasas & 0.440734930 & Regular correlación \\
\hline proteasas-ureasas & 0.688099565 & Alta correlación \\
\hline sacarasas-ureasas & 0.154676544 & Baja correlación \\
\hline
\end{tabular}

\section{Discusión}

Los parámetros del suelo biomasa microbiana total, $\mathrm{pH}$, actividad enzimática de proteasas y actividad enzimática de ureasas son sensibles a la contaminación por hidrocarburos, y pueden proveer de información valiosa al momento de realizar una evaluación.

Los resultados de variación de biomasa obtenidos para la época seca muestran un constante descenso en la cantidad de carbono microbiano disponible. Comparando este comportamiento con los resultados obtenidos para la época de lluvias y con los resultados de Fontúrbel \& Achá (2002) (obtenidos con muestras del mismo sitio, pero en época de transición), lo más probable es que este comportamiento se deba a la desecación del suelo en el laboratorio tanto de las muestras testigo como de las muestras tratadas. Sin embargo, en la muestra con hidrocarburos se observa un descenso mucho más rápido, posiblemente debido a la toxicidad de la elevada dosis (puesto que $25 \mathrm{ml} / 100 \mathrm{~g}$ es la dosis más elevada aplicada en todos los estudios, comparando con las demás dosis aplicadas de 5, 10 y $15 \mathrm{ml} / 100 \mathrm{~g})$. Tanto los resultados de biomasa correspondientes a la época de lluvias como los resultados de Fontúrbel \& Achá (2002) muestran que a menores concentraciones de contaminante $(5,10$ y $15 \mathrm{ml} / 100 \mathrm{~g})$ se observa un descenso inicial, seguido de un incremento temporal.

Estos resultados, cotejados con los estudios de diversidad y abundancia realizados paralelamente con las mismas muestras (publicaciones en preparación) se explican en términos de microorganismos biodegradadores de hidrocarburos (entre los que se incluyen 8 especies aisladas de hongos degradadorpositivo), ya que el descenso inicial de la biomasa representa la muerte de los microorganismos que no son resistentes y/o degradadores de hidrocarburos, y el incremento posterior el crecimiento y desarrollo de la porción de la comunidad que es resistente y/o capaz de degradar los contaminantes.

Estudios de Mishra et al. (2001) y Margesin \& Schinner (2001) muestran que existe un potencial de biodegradación nativo en los suelos, que puede ser incrementado por medio de bioestimulación. Mishra et al. (2001) determinaron que la cantidad de microorganismos capaces de degradar hidrocarburos encontrada naturalmente en el suelo es muy baja $\left(10^{3}\right.$ a $10^{4} \mathrm{UFC} / \mathrm{g}$ de suelo) y que demora más tiempo en degradar una misma cantidad de hidrocarburos respecto a una muestra tratada con nutrientes de bioestimulación. Las variaciones en la comunidad microbiana fueron constatadas por medio de un PCR de DNA genómico ( Mishra et al., 2001).

Margesin \& Schinner (2001) realizaron estudios comparativos de bioremediación de petróleo con y sin bioestimulación en suelos de altura (a $2875 \mathrm{~m}$ de altitud) obteniendo valores de eficiencia del $50 \pm 4 \%$ y $70 \pm 2 \%$ en suelos no tratados y tratados respectivamente. Koren et al. (2003) plantean además que la fuente de nitrógeno es uno de los principales factores limitantes del proceso de biodegradación en sistemas abiertos, especialmente del nitrógeno en forma de ácido úrico.

Por lo tanto, los resultados sugieren que este suelo tiene capacidades naturales mesurables de degradación de hidrocarburos, de acuerdo a los criterios que plantean Solano-Serena et al. (2000), Mishra et al. (2001), Margesin \& Schinner (2001) y Koren et al. (2003).

Esta capacidad natural de degradación viene regida por el principio de la infalibilidad microbiana (de acuerdo con Varnam \& Evans, 2000), que plantea que tarde o temprano, los compuestos xenobióticos del suelo serán degradados por un amplio rango de microorganismos heterotróficos (bacterias, hongos, levaduras), aunque la mayoría de las veces no se degrade el $100 \%$ del contaminante (Solano-Serena et al., 2000), puesto que la transformación de compuestos tóxicos en la naturaleza es compleja y no está regida por una sola vía bioquímica y depende de la biodisponibilidad del xenobiótico (Pieper \& Reineke, 2000). En estos casos, las bacterias juegan un papel protagónico (Varnam \& Evans, 2000), en tanto que los hongos (especialmente los filamentosos) juegan un papel integral muy importante en la bioremediación (April et al., 2000).

En cuanto a los resultados obtenidos para la variación del pH en el tiempo, es importante volver a ver las Figuras 3 y 4, en las que claramente se puede ver cómo el $\mathrm{pH}$ se regula desde una condición inicial (significativamente diferente en época seca que en 
época húmeda) hacia un rango estable de $\mathrm{pH}$ entre 5.50 y 5.60. Según Madigan et al. (1999), muchos de los microorganismos bioremediadores de hidrocarburos nativos del suelo, funcionan óptimamente a un $\mathrm{pH}$ ácido cercano a 5. Cotejando la Figuras 2, 3 y 4, se puede observar que existe una tendencia que sugiere la regulación del $\mathrm{pH}$ dentro del rango mencionado, corresponde con el crecimiento de la porción resistente y/o degradadora de la comunidad microbiana, incrementando las potencialidades de bioremediación natural de estos suelos. Así mismo, los investigadores Stapleton et al. (1998) que estudiaron la biodegradación de PAHs (hidrocarburos aromáticos policíclicos) en ambientes extremófilos con un $\mathrm{pH}$ entre 2 y 3 , también observaron que la mayoría de las especies capaces de degradar hidrocarburos no se desarrollan en condiciones de $\mathrm{pH}$ superiores a 6, por lo que se sugiere que estos grupos son predominantemente acidófilos.

Las tres enzimas estudiadas en esta investigación se escogieron teniendo en cuenta los principales procesos metabólicos del suelo, pero también en función a la disponibilidad de materiales y reactivos en la unidad de investigación (factor técnico limitante) En estudios posteriores sería recomendable considerar el estudio profundo de la actividad de las enzimas dioxigenasas, ya que son esenciales en la degradación de los hidrocarburos acíclicos (Gibson \& Parales, 2000) y tienen gran importancia en la degradación de los hidrocarburos aromáticos (Stapleton et al., 1998).

Los resultados obtenidos para la actividad enzimática de proteasas y ureasas (parámetros entre los cuales existe una alta correlación) mostraron un comportamiento muy particular: de manera coincidente con el incremento de la biomasa microbiana (aumento de la población de microorganismos degradadores) y la regulación del $\mathrm{pH}$, se observa un incremento marcado en la lisis proteica y un descenso en la actividad ureolítica. De principio, los hidrocarburos son sustancias poco solubles en agua y su estructura molecular dificulta el ataque enzimático inicial (Rittman \& McCarty, 2001), por lo que la actividad enzimática varía poco la primera semana y media, respecto al valor inicial. El incremento en la actividad de lisis proteica puede deberse fundamentalmente a la movilización y aprovechamiento de la proteína dejada por los microorganismos muertos a causa de la toxicidad de los hidrocarburos, pero también puede jugar un papel importante en la cinética molecular de los procesos de degradación (segunda y tercera semana de observación), posteriormente, cuando la proteína disponible ha sido aprovechada y ha pasado la fase más eficaz del proceso de degradación, la actividad enzimática se reduce nuevamente a niveles basales.

La actividad enzimática de las ureasas experimenta un descenso poco pronunciado durante la época crítica de la biodegradación (comparar con las Figuras 2, 3, 4 y 5), excepto por la muestra control, donde la actividad enzimática no se ve prácticamente afectada. De acuerdo a Madigan et al. (1999) y Parrish et al. (1999), uno de los factores negativos de la bioremediación y el uso de microorganismos biodegradadores es el incremento de urea en el medio. La medición de la actividad enzimática de las sacarasas no resultó sensible a este tipo de contaminación, y por lo tanto no sería un buen indicador para este caso. La similitud entre las curvas de las muestras testigo y tratadas puede deberse a que los parámetros medidos en los suelos de estudio no se ven fuertemente afectados por los hidrocarburos a concentraciones relativamente bajas.

De los resultados obtenidos y por las razones anteriormente expuestas, se plantean preliminarmente como los indicadores más apropiados para realizar una evaluación de la contaminación por hidrocarburos, la variación de biomasa, la variación del $\mathrm{pH}$ y la variación en la actividad enzimática de las proteasas, en función al tiempo. Un descenso en la biomasa (que debe ser complementado con un estudio de la diversidad de la comunidad microbiana), la regulación del $\mathrm{pH}$ en un rango de 5.00 a 5.60 (de acuerdo a referencias teóricas y experimentales), y el incremento significativo de la actividad enzimática de las proteasas, así como la magnitud de estos parámetros, son un claro indicador de los efectos tóxicos producidos por la contaminación por hidrocarburos. Adicionalmente, estos parámetros pueden ser cotejados con otros indicadores macroscópicos como la muerte y degradación de la flora, la muerte masiva de la fauna asociada (Runnalls \& Mackay, 1999) y la contaminación de cuerpos de agua relacionados directa o indirectamente con los suelos en estudio.

Las evidencias experimentales obtenidas en la presente investigación, conjuntamente con los resultados citados en Fontúrbel \& Achá (2002, 2003) muestran que los suelos estudiados tienen un gran potencial tanto de bioremediación natural in-situ como de obtención y aislamiento de microorganismos degradadores-positivo que puedan ser inoculados en otros ambientes de similares características, que pueden resultar afectados por el derrame de hidrocarburos volátiles y no volátiles, como consecuencia del transporte de hidrocarburos (Moroz, 1999; Fontúrbel \& Achá, 2000).

Si bien la degradación de hidrocarburos poca veces es del 100\% (Solano-Serena et al., 2000), la conjunción natural de diferentes especies de bacterias y hongos, con diferentes vías metabólicas (April et al., 1999; Varnam \& Evans, 2000) puede conseguir una eficiencia de más del $90 \%$, puesto que la bioremediación es un proceso propio de la naturaleza (Pieper \& Reineke, 2000). Si bien la mayor parte de los procesos de biodegradación de hidrocarburos son aeróbicos estrictos y dependen de la cantidad de oxígeno disponible en el suelo, también se han 


\section{INDICADORES DE CONTAMINACIÓN Y BIODEGRADACIÓN DE HIDROCARBUROS EN SUELOS DEL LAGO TITIKAKA}

Diciembre 2004

descrito algunas vías metabólicas anaeróbicas complejas (Parés \& Juárez, 1997), que se recomienda sean estudiadas en el futuro.

\section{Conclusiones}

Los resultados obtenidos muestran que los parámetros biomasa microbiana (en términos de carbono), pH la actividad enzimática de proteasas y ureasas, se manifiestan como buenos indicadores para la evaluación de la contaminación por hidrocarburos en suelos, y podrían ser empleados sin necesidad de realizar estudios costosos o contar con mucho equipamiento de laboratorio.

Se constató también que la actividad enzimática de las sacarasas no sería un indicador sensible para este tipo de contaminación, y por lo tanto no debería usarse en este tipo de estudios.

Los parámetros biomasa, $\mathrm{pH}$ y actividad enzimática serían también buenos indicadores de biodegradación y descontaminación de hidrocarburos en suelos, siempre y cuando sean utilizados juntos y se haga la correlación correspondiente, ya que la variación de uno solo de estos parámetros puede o no deberse a los efectos de los hidrocarburos o a alguna externalidad en el proceso, en cambio el uso combinado de estos indicadores proporciona un mejor panorama de la situación.

En base a estos estudios, se constató que los suelos de la zona del lago Titikaka con los que se trabajó tienen un cierto potencial bioremediador, puesto que poseen especies de bacterias y hongos biodegradadoras de hidrocarburos. Por esta razón estos suelos tienen un alto valor científico para el estudio y desarrollo de tecnologías económicas de bioremediación, y también tienen una relevancia significativa en el contexto socio-económico de las comunidades del lugar.

Finalmente, se constató que tanto la actividad biodegradadora como la respuesta a la contaminación son mayores en la época de lluvias que en la época seca, puesto que en la época de lluvias la humedad del ambiente favorece al crecimiento de los microorganismos en general.

\section{Agradecimientos}

A la Unidad de Suelos del Instituto de Ecología, y en especial a la Lic. Isabel Morales y a la Lic. Cristina Ruiz por apoyar esta investigación. A Sergio Coloque por el apoyo en laboratorio. A Darío Achá por su apoyo, colaboración y sus valiosos comentarios al manuscrito. Al Dr. Enrique Richard por sus valiosos comentarios al manuscrito. Al Sr. Raúl Mamani por permitirme trabajar en su propiedad para la toma de muestras.

\section{Literatura citada}

Chilón E. 1996. Manual de Edafología. Ediciones CIDAT, La Paz. : 69-83, 161-164.
Daniel W. 1996. Bioestadística: Base para el análisis de las ciencias de la salud. Editorial Limusa, México. : 832.

Duarte C. \& Ferreira S. 2000. Gasoline biodegradation in different soil microcosms. Brazilian Journal of Microbiology. 31: 45-49.

Fontúrbel F. 2002. Metodologías simplificadas para el estudio de actividad enzimática en suelos aplicadas al estudio de contaminación por hidrocarburos. Bioquímica. 2: 7-10.

Fontúrbel F. \& Achá D. 2002. Empleo del método de determinación de biomasa microbiana por fumigación-extracción para el estudio en suelos del altiplano boliviano contaminados por hidrocarburos. Ciencia Abierta Internacional 18.

Fontúrbel F. \& Achá D. 2003. Sinopsis de los estudios de bioremediación de petróleo realizados en suelos del Lago Titikaka: una alternativa social y ambientalmente viable a los problemas de contaminación por hidrocarburos. pp. 93-100. En: F. Fontúrbel \& G. García (eds.). Propuestas Para Un Desarrollo Sostenible: Lago Titikaka Por Estrategas K. Publicaciones Integrales, La Paz.

Forster J. 1995. Organic carbon. pp. 59-65. En: K. Alef \& P. Nannipieri, 1995 (eds.). Methods In Applied Soil Microbiology And Biochemistry. Academic Press, Londres.

Gibson D. \& Parales R. 2000. Aromatic hydrocarbon dioxygenases in environmental biotechnology. Current Opinion in Biotechnology. 11: 236-243.

Jenkinson D. \& Powlson S. 1976. The effects of biocidal treatments on metabolism in sol-I. Fumigation with chloroform. Soil Biol. Biochem. 8: 167-177.

Koren O., Knezevic V., Ron E. \& Rosenberg E. 2003. Petroleum pollution bioremediation using waterinsoluble uric acid as the nitrogen source. Applied and Environmental Microbiology. 69 (10): $6337-$ 6339.

Krebs C. 1985. Ecología. $2^{\circ}$ edición. Harla, México DF. : 540-542.

Madigan M., Martinko J. \& Parker J. 1999. Brock: Biología de los microorganismos. $8^{\circ}$ edición. Prentice Hall, Madrid. : 150-155, 405-408, 465, 523-524, 582-584, 698, 712-713, 774-777.

Margesin R. \& Schinner F. 2001. Bioremediation (natural attenuation and biostimulation) of dieseloil-contaminated soil in an alpine glacier skiing area. Applied and Environmental Microbiology. 67 (7): 3127-3133.

Mishra S., Jyot J.,Kuhad R. \& Lal B. 2001. Evaluation of inoculum addition to stimulate in situ bioremediation of oily-sludge-contaminated soil. Applied and Eenvironmental Microbiology. 67 (4): 1675-1681.

Morales I., Amurrio P. \& Ruiz M.C. 1997. Estudio de la salinidad en los sistemas de suka kollus y pampa 
en el transcurso de una campaña agrícola, localidad Aygachi. Ecología en Bolivia. 30: 75-89.

Moroz W.J. 1999. Contaminación del aire. pp. 492565. En: J.G. Henry \& G.W. Heinke (eds.). Ingeniería Ambiental. $2^{\circ}$ edición. Prentice Hall Pearson, México DF.

Parés R. \& Juárez A. 1997. Bioquímica de los microorganismos. Reverté, Barcelona. : 232-236.

Parrish P., Clark J. \& Prince R. 1999. Alaska Oil Spill Bioremediation Monitoring Program: An update. USEPA, NHEERL, Gulf Ecology Division, 1 Sabine Island Drive, Gulf Breeze, FL 32561. EPA.

Pieper D. \& Reineke W. 2000. Engineering bacteria for bioremediation. Current Opinion in Biotechnology. 11: 262-270.

Rittman B.E. \& McCarty P.L. 2001. Biotecnología del medio ambiente: Principios y aplicaciones. McGraw - Hill, Madrid.: 641-661.

Runnalls O.J. \& Mackay D. 2001. Crecimiento de la energía. pp. 49-84. En: J.G. Henry \& G.W. Heinke (eds.). Ingeniería Ambiental. $2^{\circ}$ edición. Prentice Hall Pearson, Mexico DF.
Smith R.L. \& Smith T.M. 2001. Ecología. $4^{\text {o }}$ edición, Addison Wesley, Madrid.

Solano-Serena F., Marchal R.,Cararégola S., Vasnier C., Lebeault J.L. \& Vandecasteele J.P. 2000. A Mycobacterium strain with extended capacities for degradation of gasoline hydrocarbons. Applied and Environmental Microbiology. 66(6): 2392-2399.

Stapleton R., Savage D., Sayler G. \& Stacey G. 1998. Biodegradation of aromatic hydrocarbons in a extremely acid environment. Applied and Environmental Microbiology. 64(11): 4180-4184.

Tate K., Ross D. \& Feltham C. 1988. A direct extraction method to estimate soil microbial $\mathrm{C}$ : effects of experimental variables and some different calibration procedures. Soil Biology and Biochemistry. 20: 329-335.

Totsche K. 1995. Quality - project design - spatial sampling. pp. 5-24 En: K. Alef \& P. Nannipieri (eds.) Methods In Applied Soil Microbiology And Biochemistry. Academic Press, Londres.

Varnam A. \& Evans M. 2000. Environmental microbiology. ASM Press, Washington. : 99-100.

\footnotetext{
${ }^{1}$ Institución donde se realizó el trabajo:

Unidad de Suelos del Instituto de Ecología de la Universidad Mayor de San Andrés

C/ 27 de Cota Cota s/n, La Paz, Bolivia.

Institución actual:

Unidad de Post-Grado Universidad Loyola

Maestría en Ingeniería de Medio Ambiente y Recursos Naturales

Av. Busch \# 1191, La Paz, Bolivia

Dirección de contacto del autor:

Casilla postal \# 180, La Paz, Bolivia

Av. 20 de Octubre \# 2005, Dep. 301, La Paz, Bolivia; Tel. +591-2-2113942

Correo electrónico: fonturbel@mbotanica.zzn.com / fonturbel@yahoo.es
} 